\title{
Comparison of Acuros (AXB) and Anisotropic Analytical Algorithm (AAA) for dose calculation in treatment of oesophageal cancer: effects on modelling tumour control probability
}

\author{
Sriram Padmanaban ${ }^{1+}$, Samantha Warren ${ }^{2 *}$, Anthony Walsh ${ }^{1}$, Mike Partridge $^{2}$ and Maria A Hawkins ${ }^{2}$
}

\begin{abstract}
Aim: To investigate systematic changes in dose arising when treatment plans optimised using the Anisotropic Analytical Algorithm (AAA) are recalculated using Acuros XB (AXB) in patients treated with definitive chemoradiotherapy (dCRT) for locally advanced oesophageal cancers.
\end{abstract}

Background: We have compared treatment plans created using AAA with those recalculated using AXB. Although the Anisotropic Analytical Algorithm (AAA) is currently more widely used in clinical routine, Acuros XB (AXB) has been shown to more accurately calculate the dose distribution, particularly in heterogeneous regions. Studies to predict clinical outcome should be based on modelling the dose delivered to the patient as accurately as possible.

Methods: $C T$ datasets from ten patients were selected for this retrospective study. VMAT (Volumetric modulated arc therapy) plans with 2 arcs, collimator rotation $\pm 5-10^{\circ}$ and dose prescription 50 Gy / 25 fractions were created using Varian Eclipse (v10.0). The initial dose calculation was performed with AAA, and AXB plans were created by re-calculating the dose distribution using the same number of monitor units $(\mathrm{MU})$ and multileaf collimator (MLC) files as the original plan. The difference in calculated dose to organs at risk (OAR) was compared using dose-volume histogram (DVH) statistics and $p$ values were calculated using the Wilcoxon signed rank test. The potential clinical effect of dosimetric differences in the gross tumour volume (GTV) was evaluated using three different TCP models from the literature.

Results: PTV Median dose was apparently 0.9 Gy lower (range: 0.5 Gy - $1.3 \mathrm{~Gy} ; \mathrm{p}<0.05$ ) for VMAT AAA plans recalculated with AXB and GTV mean dose was reduced by on average $1.0 \mathrm{~Gy}(0.3 \mathrm{~Gy}-1.5 \mathrm{~Gy} ; \mathrm{p}<0.05)$. An apparent difference in TCP of between $1.2 \%$ and $3.1 \%$ was found depending on the choice of TCP model. OAR mean dose was lower in the AXB recalculated plan than the AAA plan (on average, dose reduction: lung 1.7\%, heart 2.4\%). Similar trends were seen for CRT plans.

Conclusions: Differences in dose distribution are observed with VMAT and CRT plans recalculated with AXB particularly within soft tissue at the tumour/lung interface, where AXB has been shown to more accurately represent the true dose distribution. AAA apparently overestimates dose, particularly the PTV median dose and GTV mean dose, which could result in a difference in TCP model parameters that reaches clinical significance.

\footnotetext{
* Correspondence: samantha.warren@oncology.ox.ac.uk

${ }^{\dagger}$ Equal contributors

${ }^{2}$ CRUK/MRC Oxford Institute for Radiation Oncology, Gray Laboratories, University of Oxford, Oxford OX3 7DQ, UK

Full list of author information is available at the end of the article
} 


\section{Background}

Definitive chemoradiation (dCRT) plays an important role in the treatment of oesophageal cancer: for both squamous cell and non-operable adenocarcinoma patients, it offers a clear benefit compared to single modality treatment [1]. Recent evidence also suggests a survival benefit when CRT is used pre-operatively [2]. However, risks of local recurrence are high, with a recent study demonstrating that around $75 \%$ of recurrences in dCRT occur in the primary tumour [3], and improvement of loco-regional control appears to be a key factor in successful treatment for these patients. The introduction of advanced radiotherapy techniques, such as intensity modulation, has generated a renewed interest in dose escalation for oesophageal cancer, as delivery of a higher dose to the primary tumour, whilst maintaining dose to surrounding organs at risk at safe levels, may now be possible [4,5]. A systematic review of pre-operative CRT by Geh suggested that a radiation dose response exists for oesophageal cancer, and therefore that pathological tumour response would be improved if the radiotherapy dose were increased above $\sim 50$ Gy $[5,6]$. An investigation of the dose response would be supported by accurate knowledge of the dose delivered to the tumour. The dose delivered to normal tissues, such as lung and heart, will also be important in determining the extent of dose escalation possible for these patients.

A critical component in the analysis of the dose-response of oesophageal tumours is therefore the accuracy of the calculation of the dose distribution, which can be particularly challenging in the case of thoracic tumours, due to the presence of low density lung tissue surrounding the target volume. Advanced ('type b') dose calculation algorithms (such as AAA - Anisotropic Analytical Algorithm from Varian) now routinely available in commercial treatment planning systems show improved accuracy compared to the previous pencil beam ('type a') algorithms, but significant errors still persist at the lung/ soft tissue interface, particularly in the re-build-up region $[7,8]$. The Acuros (AXB) algorithm, recently introduced in the Eclipse treatment planning system (Varian Medical Systems, Palo Alto, USA) [9] iteratively solves the Linear Boltzman Transport Equation and has been demonstrated to show equivalent accuracy to Monte Carlo calculations in heterogeneous media [10,11]. Previous studies comparing the use of AXB vs AAA for calculating dose in patients, e.g. for breast [12], lung [13-15], and nasopharynx [16] have shown that significant differences in the calculated dose deposition may arise when the planning target volume (PTV) encompasses a range of tissue types, or is close to the interface between different density materials. Observed differences in mean PTV dose are up to $1.2 \%$ in lung [13], $1.6 \%$ in muscle [12] and $2 \%$ in bone [16], where differences in minimum PTV dose of up to $4 \%$ have been observed [16].
This study therefore seeks to compare the AXB and AAA dose calculation algorithms specifically for radiotherapy planning of thoracic oesophageal tumours, where the radiation dose in the lung/soft tissue interface region may be important in modelling both tumour control and normal tissue complications.

\section{Methods}

\section{Treatment planning}

10 patients with mid-oesophageal cancer treated with definitive chemoradiotherapy were identified retrospectively. In conformance with our institutional consent procedure, all patients prospectively gave written consent for their imaging data to be used for education and research purposes. This work was approved as part of on-going radiotherapy physics projects by the appropriate local review board. CT images with IV contrast were acquired with a slice thickness of $2.5 \mathrm{~mm}$ during 'quiet' breathing and imported into Eclipse v10 for contouring and treatment planning. Gross tumour volume (GTV) including primary tumour and nodal disease was delineated with the aid of PET/CT and endoscopic ultrasound where available. Margins of $10 \mathrm{~mm}$ axially and $20 \mathrm{~mm}$ superior/inferior were added to create the clinical target volume (CTV), and the PTV was generated by adding a $10 \mathrm{~mm}$ margin in all directions according to institutional protocol. For the studied patient subset, the mean PTV was $337 \mathrm{~cm}^{3}$ (range $140-658 \mathrm{~cm}^{3}$ ). The PTV was further divided into soft tissue 'PTV_tissue' and 'PTV_lung' where the PTV intersected with lung tissue. Normal tissues (heart, lungs, liver and cord) were delineated, with an additional planning organ at risk (CordPRV) volume created using a $5 \mathrm{~mm}$ margin around the cord.

Conformal (3D) and RapidArc (RA) treatment plans were calculated for each patient with a $2.5 \mathrm{~mm}$ isotropic dose grid, a dose prescription of $50 \mathrm{~Gy} / 25$ fractions and were initially optimised using AAA version 10.0.28 with the aim of covering $95 \%$ of PTV with the $95 \%$ isodose contour. 3D_AAA plans consisted of 3-4 beams (anterior, two lateral oblique and post), normalised to $50 \mathrm{~Gy}$ at the reference point. RA_AAA plans of 2 full arcs with collimator rotation $\pm 5-10^{\circ}$ and were normalised so that the median PTV dose was 50 Gy. AXB plans (3D_AXB and RA_AXB) were then re-calculated as dose to medium with Acuros version 10, using the same MU and MLC as for the AAA plans. The re-calculated AXB plan is believed to be a more accurate representation of the dose delivered to the patient compared to the AAA optimised treatment plans currently used in clinical routine.

\section{Plan analysis}

Dose-volume parameters for target volumes (GTV mean dose, PTV $\mathrm{D}_{95 \%}$, PTV median and PTV max dose and mean dose for PTV_Lung and PTV_tissue) were 
compared for all 10 patients. Additionally, organ at risk dose metrics (CordPRV $D_{\text {max }}$, Lung mean dose and $\mathrm{V}_{20 \mathrm{~Gy}}$, heart mean dose, $\mathrm{V}_{25 \mathrm{~Gy}}$ and $\mathrm{V}_{40 \text { Gy }}$ and liver mean dose) were recorded. The values for 3D_AAA vs 3D_AXB and RA_AAA vs RA_AXB plans were compared using the Wilcoxon signed rank test, where average differences between the paired datasets are specified as median, (range: min - range: $\max$ ) with $\mathrm{p}<0.05$ taken as significant. Tumour control probability (TCP) for the primary tumour was calculated for all plans via the differential DVH for the GTV exported in bins of $0.25 \mathrm{~Gy}$, using 3 different models taken from the literature (Figure 1).

Briefly, the Geh TCP [6] model is logistic regression fit to pathological complete response data from 26 chemoradiotherapy trials for pre-operative oesophageal cancer:

$$
T C P(z)=\frac{\exp (z)}{1+\exp (z)}
$$

and where the protocol prescribed doses of radiotherapy and chemotherapy (5FU and cisplatin) were used as variables:

$$
\begin{aligned}
z=a_{0} & +a_{1} \text { total } R T \text { dose }+a_{2} \text { total } R T \text { dose } \\
& \times \text { dose per fraction }+a_{3} \text { duration }+a_{4} \text { age } \\
& +a_{5} 5 \text { FU dose }+a_{6} \text { cisplatin dose }
\end{aligned}
$$

The Bedford TCP [17] calculation uses the WebbNahum model [18] which assumes a normal distribution of $\alpha$ the LQ parameter, with standard deviation $\sigma_{\alpha}$. Values used are $\sigma_{\alpha}=0.08 \mathrm{~Gy}^{-1}$ and $\alpha=0.40 \mathrm{~Gy}^{-1}$ chosen to fit observed local control rates for oesophageal cancer [19]. An alternative linear LQ TCP model (Isaacson [20]) using historical data (prior to the $\mathrm{CT}$ radiotherapy planning era) has also been used, although uncertainties in the dose distribution data for this model means the absolute TCP values may be less relevant to current chemoradiotherapy treatments, it is included for comparison of predicted relative differences in TCP.

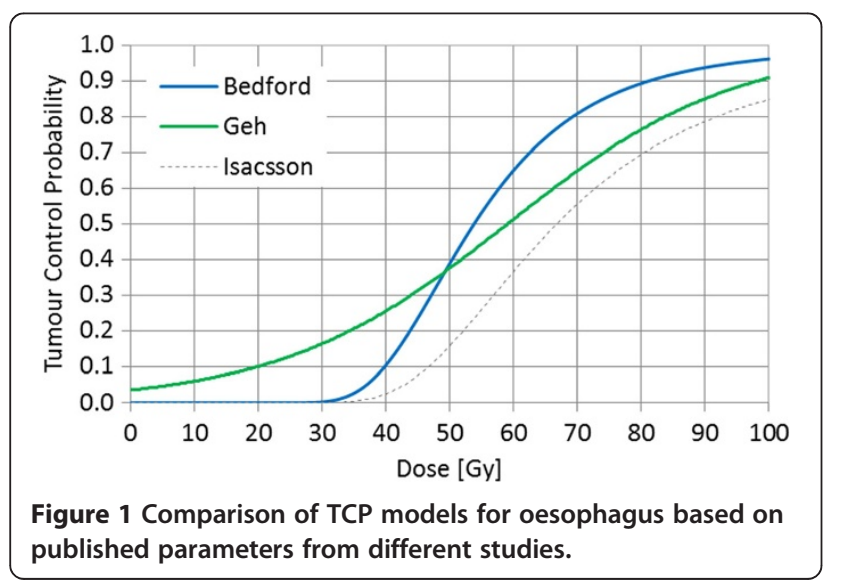

\section{Results}

\section{Dose to GTV/PTV}

A comparison of the dose distribution for RapidArc plans calculated using the AAA and AXB dose algorithms for a patient with a PTV of $529 \mathrm{~cm}^{3}$ is shown in Figure 2, indicating the lower dose obtained with the more accurate AXB dose calculation algorithm, particularly in the interior of the PTV. The differences in dose to GTV, and to the lung and tissue components of the PTV, are given in Table 1 (averaged across all ten patients). It appears that less dose is actually delivered to GTV when re-calculated using AXB, as compared to the dose predicted by AAA. For RA plans, the difference in mean dose to GTV is on average 1.0 Gy $(0.3-1.5$ Gy; $\mathrm{p}<0.05)$ and the difference in PTV median dose is on average 0.9 Gy $(0.5-1.3 \mathrm{~Gy} ; \mathrm{p}<0.05)$. As shown in Table 1 and Figure 3, the difference in calculated dose to the PTV_Lung tissue using AAA or AXB for RapidArc plans is not statistically significant. However, a difference of (on average) $0.9 \mathrm{~Gy}[0.5-1.3 \mathrm{~Gy}]$ less dose in the soft tissue region of the PTV (PTV_tissue) is found when using the $\mathrm{AXB}$ algorithm as compared to AAA. This indicates that the difference in the two dose calculation algorithms is more than a simple MU normalisation factor, and can lead to differences in relative dose distribution. Similar results for 3D conformal plans are shown in Tables 1 and 2, indicating the same apparent overestimation of dose to the soft tissue components of the target when calculated with the AAA algorithm.

\section{Dose-volume histogram analysis/OAR}

For every patient, OAR mean dose was also lower in the AXB recalculated plan than the AAA plan with the average dose difference (quoted as a percentage of the absolute value calculated with AAA) for RA plans as follows: lung $1.7 \%$, heart $2.4 \%$ (Table 2) 3D CRT plans gave similar results (Table 2). The average difference in the volume of heart receiving a moderate dose $\left(\mathrm{V}_{25} \mathrm{~Gy}\right)$ was $5.7 \%$ and the higher dose volume in the heart $\left(\mathrm{V}_{40} \mathrm{~Gy}\right)$ was also significantly less by $3.6 \%$ on average for the RA_AXB dose calculation algorithm compared to the RA_AAA plan. 3D CRT plans showed a greater difference (of up to $18.4 \%$ for one patient) for heart $\mathrm{V}_{40 \mathrm{~Gy}}$ with AXB. The CordPRV dose showed a significant reduction, on average of $3.0 \%$ for RA and $2.0 \%$ for $3 \mathrm{D}$ $\mathrm{CRT}$, when re-calculated using the AXB dose algorithm to obtain a more accurate representation of the dose truly delivered to each patient.

\section{TCP analysis}

An apparent difference in TCP expressed here as median [range: min, range: max] was found for all patients when re-calculating plans using $\mathrm{AXB}$, due to the apparent overestimation of GTV dose by AAA. The magnitude of 

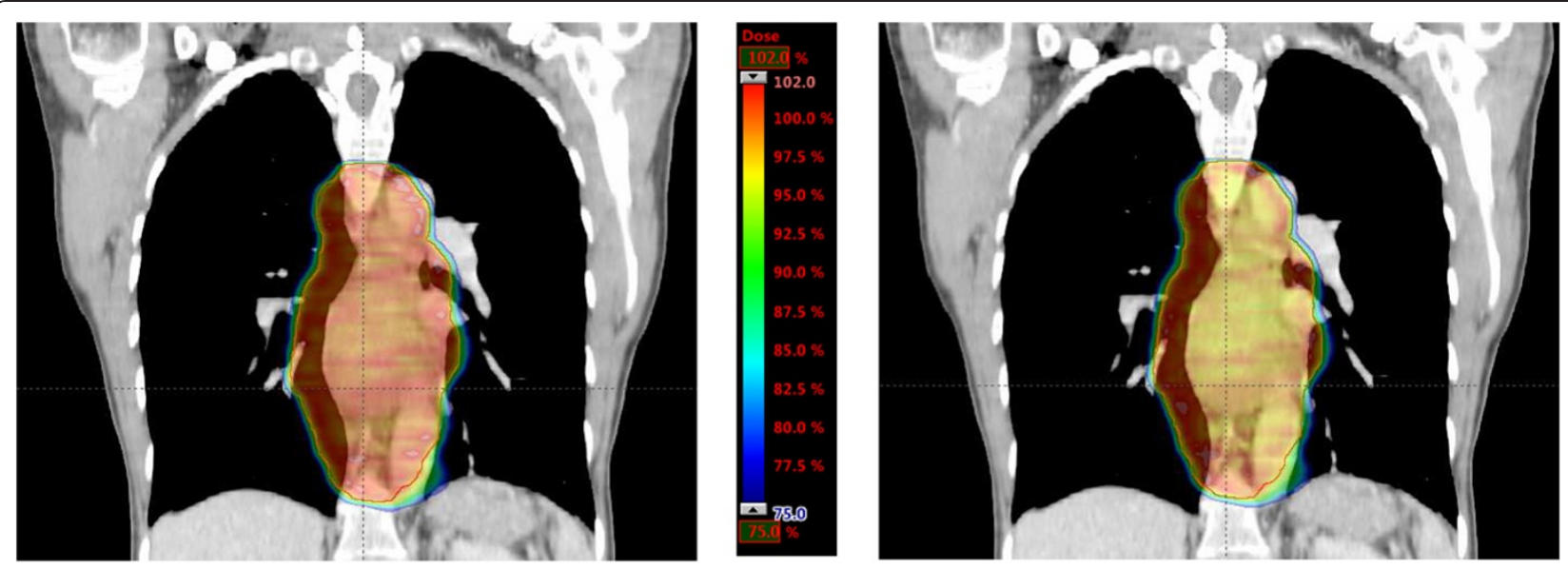

Figure 2 Comparison of dose distribution calculated using AAA and AXB dose calculation algorithms for mid-oesophageal cancer. Left: Coronal slice of RapidArc plan calculated using AAA showing the dose distribution for a mid-oesophageal cancer patient with the largest proportion of lung tissue in the PTV (red contour, total size of $529 \mathrm{~cm} 3$ ). The dose colour scale ranges from 75\% (blue) to 102\% (red) of the prescribed dose of $50 \mathrm{~Gy}$. Right: The same RapidArc plan recalculated using the AXB dose calculation algorithm (for the same patient, with the same dose scale) showing a reduction in dose, particularly in soft tissue in the interior of the PTV.

this difference depended on the choice of TCP model. Geh predicted a $1.2 \%[-0.4 \%,+1.9 \%]$ difference in local control from (RA_AAA) to (RA_AXB) $(\mathrm{p}<0.05)$, and the Bedford TCP model predicts a reduction of $3 \%$ $[1.1 \%, 4.5 \%]$ from (RA_AAA) to (RA_AXB) $(\mathrm{p}<0.05)$. Similar values are seen for the 3D CRT plans: TCP Geh

Table 1 Comparison of target volume dose calculated using AAA or AXB for all patients for a) RapidArc plans and b) 3D conformal radiotherapy (CRT) plans

\begin{tabular}{|c|c|c|c|}
\hline \multicolumn{3}{|l|}{ a) } & \multirow{4}{*}{ p } \\
\hline \multirow[t]{3}{*}{ Target (dose metric) } & \multicolumn{2}{|c|}{ Mean dose $[\min , \max ]$ in Gy } & \\
\hline & RA_AAA & RA_AXB & \\
\hline & CRT_AAA & CRT_AXB & \\
\hline GTV (mean) & $49.9[49.6,50.0]$ & $48.8[48.3,49.6]$ & 0.01 \\
\hline PTV $\left(D_{95 \%}\right)$ & $48.7[48.0,49.1]$ & $47.7[47.1,48.2]$ & 0.01 \\
\hline PTV (median) & 50.0 (normalisation) & $49.1[48.7,49.5]$ & 0.01 \\
\hline PTV (max) & $52.3[51.5,53.1]$ & $53.1[52.5,53.9]$ & 0.01 \\
\hline PTV_Lung (mean) & $49.6[49.2,49.8]$ & $49.7[49.0,50.1]$ & 0.18 \\
\hline PTV_tissue (mean) & $49.9[49.8,50.0]$ & $49.0[48.7,49.5]$ & 0.01 \\
\hline \multicolumn{4}{|l|}{ b) } \\
\hline \multirow[t]{2}{*}{ Target (dose metric) } & \multicolumn{2}{|c|}{ Mean dose $[\min , \max ]$ in Gy } & p \\
\hline & CRT_AAA & CRT_AXB & \\
\hline GTV (mean) & $50.6[49.8,51.2]$ & $49.6[49.0,50.1]$ & 0.01 \\
\hline PTV (D95\%) & $48.2[45.7,49.4]$ & $47.4[44.9,48.6]$ & 0.01 \\
\hline PTV (median) & $50.2[49.6,50.7]$ & $49.2[48.1,49.7]$ & 0.01 \\
\hline Norm point & 50.0 (normalisation) & $48.8[48.0,49.7]$ & \\
\hline PTV (max) & $52.9[51.9,53.8]$ & $53.4[52.4,54.8]$ & 0.01 \\
\hline PTV_Lung (mean) & $49.4[47.8,50.3]$ & $49.4[47.7,50.7]$ & 0.12 \\
\hline PTV_tissue (mean) & $50.2[49.5,50.7]$ & $49.2[48.4,49.8]$ & 0.01 \\
\hline
\end{tabular}

(CRT_AXB) is $1.4 \%[0.5 \%, 1.9 \%]$ different from the CRT_AAA mean TCP and Bedford CRT_AAA mean TCP changes by $3.1 \%$ [1.3\%, 4.3\%]. The Isacsson model TCP was $1.8 \%$ [0.7\%, 2.7\%] lower for both RA and CRT plans when comparing AXB to the AAA dose calculation algorithm.

\section{Discussion}

This study compared the dose distributions obtained by two different dose calculation algorithms (AXB and AAA) for mid-oesophageal tumours. Lower doses were found when re-calculating AAA treatment plans with the AXB algorithm, and this dose difference was clinically significant for the GTV, PTV and certain organs at risk (heart, cord). The lower doses predicted by AXB

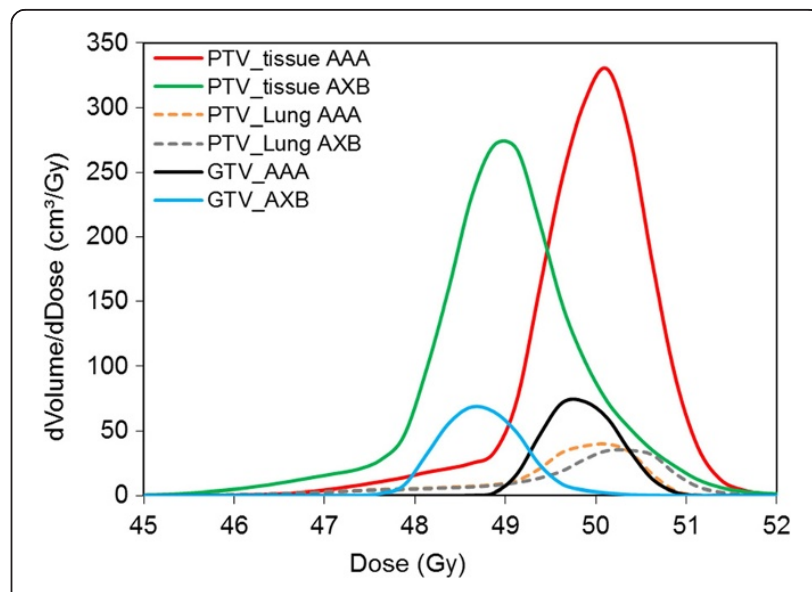

Figure 3 Differential DVH for GTV and PTV using AAA and AXB dose calculation algorithms. 
Table 2 Mean \% dose difference (quoted as a percentage of the absolute value calculated with AAA) to organs at risk calculated using AAA or AXB for a) RapidArc plans and b) 3D conformal radiotherapy (CRT) plans

a)

\begin{tabular}{lll} 
OAR and dose metric & $\begin{array}{l}\text { Mean difference [min, max }] \\
\text { \% (RA_AAA) - (RA_AXB) }\end{array}$ & p \\
\hline Cord PRV D $\max$ & $-3.0 \%[-3.9 \%,-1.6 \%]$ & $<0.05$ \\
Lung Mean & $-1.7 \%[-5.2 \%,-0.4 \%]$ & $<0.05$ \\
Lung $V_{20 G y}$ & $-2.7 \%[-8.3 \%, 0.0 \%]$ & $<0.05$ \\
Heart Mean & $-2.4 \%[-3.0 \%,-1.2 \%]$ & $<0.05$ \\
Heart $V_{25 G y}$ & $-5.7 \%[-7.4 \%,-4.5 \%]$ & $<0.05$ \\
Heart $V_{40 G y}$ & $-3.6 \%[-8.3 \%,-1.3 \%]$ & $<0.05$ \\
Liver Mean & $-1.2 \%[-2.2 \%, 0.0 \%]$ & NS \\
\hline
\end{tabular}

b)

\begin{tabular}{lll} 
OAR and dose metric & $\begin{array}{l}\text { Mean difference [min, max }] \\
\text { \% (CRT_AAA) }-(\text { CRT_AXB) }\end{array}$ & p \\
\hline Cord PRV $D_{\max }$ & $-2.0 \%[-8.2 \%, 2.7 \%]$ & $<0.05$ \\
Lung Mean & $-2.2 \%[-4.5 \%, 0.3 \%]$ & $<0.05$ \\
Lung $V_{20 G y}$ & $-1.5 \%[-3.7 \%, 0.4 \%]$ & $<0.05$ \\
Heart Mean & $-2.6 \%[-3.4 \%,-1.7 \%]$ & $<0.05$ \\
Heart $V_{25 G y}$ & $-3.0 \%[-12.4 \%,-0.3 \%]$ & $<0.05$ \\
Heart $V_{40 G y}$ & $-6.9 \%[-18.4 \%,-2.2 \%]$ & $<0.05$ \\
Liver Mean & $-1.5 \%[-2.5 \%, 0.6 \%]$ & NS \\
\hline
\end{tabular}

compared to AAA are found for both 3D conformal and RapidArc plans, indicating that the differences in dose distribution for oesophageal cancer are not dependent on the beam delivery technique. This is in line with results from the literature, for example for lung tumours, where AXB predicts up to 1.1 Gy less than AAA to the soft tissue in the PTV for 3D, IMRT and RA plans [13]. Previous studies investigating the use of AXB in heterogeneous media $[10,11,14]$ suggest that this algorithm is more accurate than the widely-used AAA, especially at the lung/soft tissue interface region [13,21,22]. In particular, AXB shows a much better agreement with Monte Carlo calculations [23] or dose measurements [24] in regions of re-buildup in soft tissue after the beam has passed through low density tissue such as lung or air. Our AXB results can therefore be considered as a better approximation of the true dose distribution than data obtained using AAA, and indicate an apparent overestimation of dose to the primary tumour of around $1 \mathrm{~Gy}$ when calculated with AAA. The location of the dose over-estimation is particularly pertinent in the case of oesophageal cancers, where most local recurrences occur within the GTV, well inside the treatment field.

However, as most clinical outcome data is based on dose distribution calculated using "type b" (AAA) or "type a" (pencil beam) dose algorithms, the interpretation of these results in the context of current radiotherapy treatments and future dose escalation trials should be carefully analysed. Normalisation of 3D CRT radiotherapy treatment plans using $\mathrm{AXB}$ to meet the protocol dose prescription of 50 Gy would result in an increase in MU of around 2\% (range $1.0 \%$ to $2.8 \%$ ), with a corresponding increase in mean dose delivered to the surrounding normal tissues contained within the Body-PTV contour.

Alternative methods to account for the differences in dose distribution include creation of algorithm-specific radiobiological parameters, such as TCP models of local control in breast cancer with specific pencil beam, AAA or AXB parameters [25]; or re-analysis of the fitting parameters to take into account the improved dose calculation. This latter approach has been used in a study of dose-volume effects in radiation pneumonitis used for NTCP modelling of toxicity in lung [26]. The absolute TCP values from the Geh and Bedford models were broadly consistent at $\sim 37 \%$. The smaller TCP difference predicted by the Geh model ( $1.2 \%$ vs. $3 \%$ for Bedford) is likely to be due to the fact that the Geh model was derived from more heterogeneous population data.

It should be noted that optimisation of RA plans using AXB will, or course, produce plans which meet the prescribed dose constraints, but have a different MLC fluence. We have shown that this is not simply a scaled version of the AAA result (data not shown). One limitation of our current study is that we have compared dose distributions calculated using the planning CT dataset acquired during free-breathing. Studies using 4D-CT scans for dose accumulation throughout the respiratory cycle suggest that the dosimetric effect of movement is slight, even for small tumours entirely surrounded by low density lung [27]. The cranial-caudal location of the primary tumour in mid-oesophageal cancers can also be difficult to identify, although an analysis of fiducial marker movement during 4D-CT scans for mid-oesophageal cancer suggested $95 \%$ of intra-fractional tumour movement would be covered by a $7.4 \mathrm{~mm}$ margin in the cranialcaudal direction, and this is within our CTV to PTV margin of $10 \mathrm{~mm}$. Variations in inter-fractional displacement have also been investigated using repeated 4D-CT scans [28], and indicate a general trend for tumour regression during the course of radiotherapy, but no systematic displacement was observed for mid-oesophageal tumours even after 20 fractions of radiotherapy treatment.

\section{Conclusions}

Recalculating treatment plans for mid-oesophageal cancer using the more accurate AXB dose calculation algorithm instead of AAA produces a lower dose to the PTV and GTV, and lower mean dose to the surrounding organs at risk. The difference in dose is largest in areas of soft tissue surrounded by low density lung (such as the oesophageal primary tumour volume and the heart). 
Modelling dose-response from clinical trial data should specify the dose calculation algorithm used, in order to avoid bias in the generation of dose-volume constraints and radiobiological model parameters.

\section{Competing interests}

The authors declare that they have no competing interests.

\section{Authors' contributions}

SP carried out the planning study and data analysis and drafted the manuscript. SW conceived of the study, participated in its design and data analysis, and drafted the manuscript. AW participated in the planning study and data analysis. MP provided radiobiological modelling of the data and participated in the study design and drafting of the manuscript. MH participated in the design of the study and drafting of the manuscript and provided clinical expertise. All authors read and approved the final manuscript.

\section{Acknowledgements}

The authors gratefully acknowledge funding from Cancer Research UK and the UK Medical Research Council grant references C5255/A15935 and MC_PC_12001/2.

\section{Author details}

'Oxford Cancer Centre, Oxford University Hospitals, Oxford OX3 7LE, UK. ${ }^{2}$ CRUK/MRC Oxford Institute for Radiation Oncology, Gray Laboratories, University of Oxford, Oxford OX3 7DQ, UK.

Received: 6 May 2014 Accepted: 3 December 2014

Published online: 23 December 2014

\section{References}

1. Cooper JS, Guo MD, Herskovic A, Macdonald JS, Martenson JA Jr, Al-Sarraf M, Byhardt R, Russell AH, Beitler JJ, Spencer S, Asbell SO, Graham MV, Leichman LL: Chemoradiotherapy of locally advanced esophageal cancer: long-term follow-up of a prospective randomized trial (RTOG 85-01). Radiation therapy oncology group. JAMA 1999, 281(17):1623-1627.

2. van Hagen $P$, Hulshof MC, van Lanschot JJ, Steyerberg EW, van Berge Henegouwen MI, Wijnhoven BP, Richel DJ, Nieuwenhuijzen GA, Hospers GA, Bonenkamp JJ, Cuesta MA, Blaisse RJ, Busch OR, ten Kate FJ, Creemers GJ, Punt CJ, Plukker JT, Verheul HM, Spillenaar Bilgen EJ, van Dekken H, van der Sangen MJ, Rozema T, Biermann K, Beukema JC, Piet AH, van Rij CM, Reinders JG, Tilanus HW, van der Gaast A: Preoperative chemoradiotherapy for esophageal or junctional cancer. N Engl J Med 2012, 366(22):2074-2084.

3. Welsh J, Settle SH, Amini A, Xiao L, Suzuki A, Hayashi Y, Hofstetter W, Komaki R, Liao Z, Ajani JA: Failure patterns in patients with esophageal cancer treated with definitive chemoradiation. Cancer 2012, 118(10):2632-2640.

4. Welsh J, Palmer MB, Ajani JA, Liao Z, Swisher SG, Hofstetter WL, Allen PK, Settle SH, Gomez D, Likhacheva A, Cox JD, Komaki R: Esophageal cancer dose escalation using a simultaneous integrated boost technique. Int $J$ Radiat Oncol Biol Phys 2012, 82(1):468-474.

5. Warren S, Partridge M, Carrington R, Hurt C, Crosby T, Hawkins MA: Radiobiological determination of dose escalation and normal tissue toxicity in definitive chemoradiation therapy for esophageal cancer. Int J Radiat Oncol Biol Physics 2014, 90(2):423-429.

6. Geh JI, Bond SJ, Bentzen SM, Glynne Jones R: Systematic overview of preoperative (neoadjuvant) chemoradiotherapy trials in oesophageal cancer: evidence of a radiation and chemotherapy dose response. Radiother Oncol 2006, 78(3):236-244.

7. Van Esch A, Tillikainen L, Pyykkonen J, Tenhunen M, Helminen H, Siljamaki S, Alakuijala J, Paiusco M, Lori M, Huyskens DP: Testing of the analytical anisotropic algorithm for photon dose calculation. Med Phys 2006, 4(11):130-148

8. Aarup LR, Nahum AE, Zacharatou C, Juhler-Nottrup T, Knoos T, Nystrom H, Specht L, Wieslander E, Korreman SS: The effect of different lung densities on the accuracy of various radiotherapy dose calculation methods: implications for tumour coverage. Radiother Oncol 2009, 91(3):405-414.

9. Vassiliev ON, Wareing TA, McGhee J, Failla G, Salehpour MR, Mourtada F: Validation of a new grid-based Boltzmann equation solver for dose calculation in radiotherapy with photon beams. Phys Med Biol 2010, 55(3):581-598.
10. Fogliata A, Nicolini G, Clivio A, Vanetti E, Cozzi L: Dosimetric evaluation of Acuros $\mathrm{XB}$ advanced dose calculation algorithm in heterogeneous media. Radiat Oncol 2011, 6:82.

11. Bush K, Gagne IM, Zavgorodni S, Ansbacher W, Beckham W: Dosimetric validation of Acuros XB with monte carlo methods for photon dose calculations. Med Phys 2011, 38(4):2208-2221.

12. Fogliata A, Nicolini G, Clivio A, Vanetti E, Cozzi L: On the dosimetric impact of inhomogeneity management in the Acuros XB algorithm for breast treatment. Radiat Oncol 2011, 6:103.

13. Fogliata A, Nicolini G, Clivio A, Vanetti E, Cozzi L: Critical appraisal of Acuros $\mathrm{XB}$ and anisotropic analytic algorithm dose calculation in advanced non-small-cell lung cancer treatments. Int J Radiat Oncol Biol Phys 2012, 83(5):1587-1595.

14. Kroon PS, Hol S, Essers M: Dosimetric accuracy and clinical quality of Acuros $\mathrm{XB}$ and $\mathrm{AAA}$ dose calculation algorithm for stereotactic and conventional lung volumetric modulated arc therapy plans. Radiat Oncol 2013, 8(1):149.

15. Liu HW, Nugent Z, Clayton R, Dunscombe P, Lau H, Khan R: Clinical impact of using the deterministic patient dose calculation algorithm Acuros XB for lung stereotactic body radiation therapy. Acta Oncol 2013.

16. Kan MW, Leung LH, Yu PK: Dosimetric impact of using the Acuros XB algorithm for intensity modulated radiation therapy and RapidArc planning in nasopharyngeal carcinomas. Int J Radiat Oncol Biol Phys 2013, 85(1):e73-80.

17. Bedford JL, Viviers L, Guzel Z, Childs PJ, Webb S, Tait DM: A quantitative treatment planning study evaluating the potential of dose escalation in conformal radiotherapy of the oesophagus. Radiother Oncol 2000, 57(2):183-193.

18. Webb S, Nahum AE: A model for calculating tumour control probability in radiotherapy including the effects of inhomogeneous distributions of dose and clonogenic cell density. Phys Med Biol 1993, 38(6):653-666.

19. al-Sarraf M, Martz K, Herskovic A, Leichman L, Brindle JS, Vaitkevicius VK, Cooper J, Byhardt R, Davis L, Emami B: Progress report of combined chemoradiotherapy versus radiotherapy alone in patients with esophageal cancer: an intergroup study. J Clin Oncol 1997, 15(1):277-284.

20. Isacsson U, Lennernas B, Grusell E, Jung B, Montelius A, Glimelius B: Comparative treatment planning between proton and $x$-ray therapy in esophageal cancer. Int J Radiat Oncol Biol Phys 1998, 41(2):441-450.

21. Kathirvel M, Subramanian S, Clivio A, Arun G, Fogliata A, Nicolini G, Subramani V, Thirumalai Swamy S, Vanetti E, Cozzi L: Critical appraisal of the accuracy of Acuros-XB and anisotropic analytical algorithm compared to measurement and calculations with the compass system in the delivery of rapidArc clinical plans. Radiat Oncol 2013, 8(1):140.

22. Han T, Mikell JK, Salehpour M, Mourtada F: Dosimetric impact of Acuros XB deterministic radiation transport algorithm for heterogeneous dose calculation in lung cancer. Med Phys 2013, 40(5):051710.

23. Han T, Mikell JK, Salehpour M, Mourtada F: Dosimetric comparison of Acuros XB deterministic radiation transport method with Monte Carlo and model-based convolution methods in heterogeneous media. Med Phys 2011, 38(5):2651-2664.

24. Hoffmann L, Jorgensen MB, Muren LP, Petersen JB: Clinical validation of the Acuros XB photon dose calculation algorithm, a grid-based Boltzmann equation solver. Acta Oncol 2012, 51(3):376-385.

25. Petillon SA, Swinnen G, Defraene K, Verhoeven C, Weltens F, Van Der H: The photon dose calculation algorithm used in breast radiotherapy has significant impact on the parameters of the radiobiological models. J Appl Clin Med Physics 2014. accepted.

26. De Jaeger K, Hoogeman MS, Engelsman M, Seppenwoolde Y, Damen EM, Mijnheer BJ, Boersma $\sqcup$, Lebesque JV: Incorporating an improved dosecalculation algorithm in conformal radiotherapy of lung cancer: re-evaluation of dose in normal lung tissue. Radiother Oncol 2003, 69(1):1-10.

27. Admiraal MA, Schuring D, Hurkmans CW: Dose calculations accounting for breathing motion in stereotactic lung radiotherapy based on $4 \mathrm{D}-\mathrm{CT}$ and the internal target volume. Radiother Oncol 2008, 86(1):55-60.

28. Wang JZ, Li JB, Wang W, Qi HP, Ma ZF, Zhang YJ, Fan TY, Shao Q, Xu M: Detection of interfraction displacement and volume variance during radiotherapy of primary thoracic esophageal cancer based on repeated four-dimensional CT scans. Radiat Oncol 2013, 8:224. 